\title{
The Cartesian product of cycles with small 2-rainbow domination number
}

\author{
Zofia Stępień • Lucjan Szymaszkiewicz • \\ Maciej Zwierzchowski
}

(C) The Author(s) 2013. This article is published with open access at Springerlink.com

\begin{abstract}
The concept of 2-rainbow domination of a graph $G$ coincides with the ordinary domination of the prism $G \square K_{2}$ (see Brešar et al., Taiwan J Math 12:213$225,2008)$. Hence $\gamma_{r 2}\left(C_{m} \square C_{n}\right) \geq \frac{m n}{3}$. In this paper we give full characterization of graphs $C_{m} \square C_{n}$ with $\gamma_{r 2}\left(C_{m} \square C_{n}\right)=\frac{m n}{3}$.
\end{abstract}

Keywords Domination - Rainbow domination - Cartesian product of graphs

Mathematics Subject Classification 05C69

\section{Introduction}

For notation and graph theory terminology not given here, we follow Diestel (1997) and Haynes et al. (1998). Let $G=(V(G), E(G))$ be a finite, simple and undirected graph with vertex set $V(G)$ and edge set $E(G)$. The open neighborhood of a vertex $v$ is $N(v)=\{u \in V(G): u v \in E(G)\}$. If $A \subset V(G)$, then $N(A)$ denotes the union of open neighborhoods of all vertices of $A$. For two subsets $A, B$ of $V(G)$, $E(A, B)=\{a b \in E(G): a \in A, b \in B\}$.

\footnotetext{
Z. Stępień · M. Zwierzchowski ( $\square)$

School of Mathematics, West Pomeranian University of Technology,

al. Piastów 48/49, 70-310 Szczecin, Poland

e-mail: mzwierz@zut.edu.pl

Z. Stępień

e-mail: stepien@zut.edu.pl
}

L. Szymaszkiewicz

Institute of Mathematics, Szczecin University, Wielkopolska 15,

70-451 Szczecin, Poland

e-mail: lucjansz@wmf.univ.szczecin.pl 
The Cartesian product $G \square H$ of graphs $G$ and $H$ is the graph with vertex set $V(G) \times V(H)$, where two vertices are adjacent if and only if they are equal in one coordinate and adjacent in the other.

We restrict our attention to the Cartesian product of $C_{n}$ and $C_{m}, n, m \geq 3$. Let $V\left(C_{n}\right)=\{0,1, \ldots, n-1\}, E\left(C_{n}\right)=\{i(i+1),(n-1) 0: i=0,1, \ldots, n-2\}$. Hence we will denote vertices of $V\left(C_{m} \square C_{n}\right)$ by $(i, j)$ for $i=0,1, \ldots, m-1$ and $j=0,1, \ldots, n-1$. For the arbitrary integers $i$ and $j$ we will use the following notation

$$
[i, j]=(i \bmod m, j \bmod n) .
$$

A function $f: V(G) \rightarrow \mathscr{P}(\{1, \ldots, k\})$ is called a $k$-rainbow dominating function of $G$ (for short $k R D F$ of $G$ ) if $\bigcup_{u \in N(v)} f(u)=\{1, \ldots, k\}$, for each vertex $v \in V(G)$ with $f(v)=\varnothing$. By $w(f)$ we mean $\sum_{v \in V(G)}|f(v)|$ and we call it the weight of a function $f$ in $G$. The minimum weight of a $k R D F$ of $G$ is called the k-rainbow domination number of $G$ and it is denoted by $\gamma_{r k}(G)$. If $f$ is a $k R D F$ of $G$ and $w(f)=\gamma_{r k}(G)$, then $f$ is called a $\gamma_{r k}$-function. For more information about rainbow domination we refer the reader to Brešar and Šumenjak (2007), Tong et al. (2009), Hartnell and Rall (1998), Wu and Rad (2013), Wu and Xing (2010), Šumenjak et al. (2013) and Xu (2009), where authors consider, in particular, connections between rainbow domination and Vizing conjecture.

Let $f$ be any $2 R D F$ of $C_{m} \square C_{n}$. Define the following sets

$$
\begin{aligned}
V_{0} & =\left\{v \in V\left(C_{m} \square C_{n}\right): f(v)=\varnothing\right\}, \\
V_{1} & =\left\{v \in V\left(C_{m} \square C_{n}\right): f(v)=\{1\} \text { or } f(v)=\{2\}\right\}, \\
V_{2} & =\left\{v \in V\left(C_{m} \square C_{n}\right): f(v)=\{1,2\}\right\}, \\
V_{i_{1} i_{2}} & =\left\{v \in V_{0}:\left|N(v) \cap V_{t}\right|=i_{t}, t=1,2\right\}, \\
E_{1} & =\left\{u v \in E\left(C_{m} \square C_{n}\right): u, v \in V_{1}\right\}, \\
E_{2} & =\left\{u v \in E\left(C_{m} \square C_{n}\right): u, v \in V_{2}\right\}, \\
E_{12} & =\left\{u v \in E\left(C_{m} \square C_{n}\right): u \in V_{1}, v \in V_{2}\right\} .
\end{aligned}
$$

We need the following technical lemma.

Lemma 1 (Stępień and Zwierzchowski 2012) Let $f$ be any $2 R D F$ of $C_{m} \square C_{n}$. Then

$$
w(f)=\frac{m n}{3}+\frac{\beta}{6},
$$

where

$$
\begin{aligned}
\beta= & 2\left|V_{2}\right|+\left|V_{11}\right|+3\left|V_{12}\right|+5\left|V_{13}\right|+2\left|V_{21}\right|+4\left|V_{22}\right|+\left|V_{30}\right| \\
& +3\left|V_{31}\right|+2\left|V_{40}\right|+2\left|V_{02}\right|+4\left|V_{03}\right|+6\left|V_{04}\right| \\
& +3\left|E_{12}\right|+2\left|E_{1}\right|+4\left|E_{2}\right| .
\end{aligned}
$$

Corollary 1 Let $f$ be any $2 R D F$ of $C_{m} \square C_{n}$. Then $w(f) \geq \frac{m n}{3}$ and equality holds if and only if $\beta=0$.

In this paper we will use the following form of the Chinese Reminder Theorem. 
Theorem 1 (Chinese Reminder Theorem) Two simultaneous congruences

$$
\begin{array}{ll}
x \equiv a & (\bmod m), \\
x \equiv b & (\bmod n)
\end{array}
$$

are solvable if and only if $a \equiv b(\bmod \operatorname{gcd}(m, n))$. Moreover the solution is unique modulo $\operatorname{lcm}(m, n)$.

\section{Results}

For any integer $s$, let $L_{s}=\left\{[k, k-s] \in V\left(C_{m} \square C_{n}\right): k=0, \pm 1, \pm 2, \ldots\right\}$. The following theorem is a consequence of the Chinese Reminder Theorem.

Theorem 2 We have

$$
V\left(C_{m} \square C_{n}\right)=\bigcup_{s=0}^{\operatorname{gcd}(m, n)-1} L_{s} .
$$

The sum is disjoint and $\left|L_{s}\right|=\operatorname{lcm}(m, n)$.

Proof By definition of $L_{s}$ we have $\bigcup_{s=0}^{\operatorname{gcd}(m, n)-1} L_{s} \subseteq V\left(C_{m} \square C_{n}\right)$. Let $(i, j) \in$ $V\left(C_{m} \square C_{n}\right)$ and let $s \in\{0,1, \ldots, \operatorname{gcd}(m, n)-1\}$ be such that $s \equiv i-j$ $(\bmod \operatorname{gcd}(m, n))$. By Theorem 1 there exists an integer $k$ such that

$$
\begin{aligned}
& k \equiv i \quad(\bmod m), \\
& k \equiv j+s \quad(\bmod n) .
\end{aligned}
$$

Consequently, $(i, j)=[k, k-s] \in L_{s}$.

Next if the above system has any solution for a fixed $(i, j)$ and some $s$, then again by Theorem 1 we have $s \equiv i-j(\bmod \operatorname{gcd}(m, n))$. Hence $L_{s_{1}} \cap L_{s_{2}}=\emptyset$ for $s_{1} \neq s_{2}$ and $s_{1}, s_{2} \in\{0,1, \ldots, \operatorname{gcd}(m, n)-1\}$. Finally, observe that cardinality of $L_{s}$ is the same for each $s$. Therefore $\left|L_{s}\right|=\operatorname{lcm}(m, n)$.

For any integer $s$, let us denote

$$
\llbracket s \rrbracket=s \bmod \operatorname{gcd}(m, n) .
$$

Corollary 2 The following holds:

1. for any integers $i, j$ we have $[i, j] \in L_{\llbracket i-j \rrbracket}$,

2. if $\operatorname{gcd}(m, n)>1$, then for any integer $s$ we have

$$
N\left(L_{\llbracket s \rrbracket}\right)=L_{\llbracket s-1 \rrbracket} \cup L_{\llbracket s+1 \rrbracket},
$$

3. if $\operatorname{gcd}(m, n)=1$, then we have

$$
V\left(C_{m} \square C_{n}\right)=L_{0} .
$$


Now we introduce some definitions. Let $f$ be a 2-rainbow dominating function of $C_{m} \square C_{n}$. We say that $f$ is positive if for any $(i, j) \in V\left(C_{m} \square C_{n}\right)$ the following implication holds:

$$
(i, j) \in V_{1} \Rightarrow L_{\llbracket i-j \rrbracket} \subset V_{1}
$$

Let $L_{s}^{-}=\left\{[k,-k+s] \in V\left(C_{m} \square C_{n}\right): k=0, \pm 1, \pm 2, \ldots\right\}$. Note that $[i, j] \in$ $L_{\llbracket i+j \rrbracket}^{-}$. We say that $f$ is negative if for any $(i, j) \in V\left(C_{m} \square C_{n}\right)$ the following implication holds:

$$
(i, j) \in V_{1} \Rightarrow L_{\llbracket i+j \rrbracket}^{-} \subset V_{1}
$$

Lemma 2 Let $f$ be a 2-rainbow dominating function of $C_{m} \square C_{n}$ such that $w(f)=$ $\frac{m n}{3}$. Then $f$ is either positive or negative.

Proof Let $f$ be a 2-rainbow dominating function of $C_{m} \square C_{n}$ such that $w(f)=\frac{m n}{3}$. By Corollary 1 , we get $\beta=0$. Hence $\left|V_{2}\right|=\left|E_{1}\right|=\left|V_{30}\right|=\left|V_{40}\right|=0$.

Take any vertex $(i, j) \in C_{m} \square C_{n}$ such that $(i, j) \in V_{1}$. Assume, without loss of generality, that $f((i, j))=\{1\}$. Since $\left|E_{1}\right|=0$, we have

$$
N((i, j)) \subset V_{0}
$$

We claim that also

$$
\{[i-2, j],[i+2, j],[i, j-2],[i, j+2]\} \subset V_{0} .
$$

To prove (2) suppose the contrary: assume, without loss of generality, that $[i, j+2] \in$ $V_{1}$. Then $[i+1, j+1],[i+1, j+2] \in V_{0}$ (otherwise $\left|V_{30} \cup V_{40} \cup E_{1}\right| \neq 0$ ). Since the vertex $[i+1, j+1]$ must be dominated in the sense of 2-rainbow domination, we get $\left|V_{2}\right| \neq 0$, a contradiction.

Observe that exactly one of $[i+1, j+1],[i+1, j-1]$ belongs to $V_{1}$. Indeed, on the one hand at most one of $[i+1, j+1],[i+1, j-1]$ belongs to $V_{1}$, since otherwise $\left|V_{30}\right| \neq 0$. On the other hand at least one of $[i+1, j+1],[i+1, j-1]$ belongs to $V_{1}$ and $f([i+1, j+1])=\{2\}$ or $f([i+1, j-1])=\{2\}$ (otherwise $[i+1, j]$ would not be dominated in the sense of 2-rainbow domination). Thus either $f([i+1, j+1])=\{2\}$ or $f([i+1, j-1])=\{2\}$.

Assume that $f([i+1, j+1])=\{2\}$. This assumption combined with (1) and (2) imply that $[i+2, j+2] \in V_{1}$. By induction we get $L_{[[i-j]]} \subset V_{1}$. Similarly, if $f([i+1, j-1])=\{2\}$, then we get $L_{[[i+j]]}^{-} \subset V_{1}$. We have shown that if $(i, j) \in V_{1}$, then either $L_{[[i-j]]} \subset V_{1}$ or $L_{[[i+j]]}^{-} \subset V_{1}$. Thus $f$ is positive or negative.

Finally, suppose that $f$ is positive and negative. This means that $L_{s_{1}} \subset V_{1}$ and $L_{s_{2}}^{-} \subset V_{1}$ for $s_{1}, s_{2} \in\{0,1, \ldots, \operatorname{gcd}(m, n)-1\}$. To eliminate this possiblity, we will consider the following two cases.

(a) There exists $k$ such that $s_{1}+s_{2} \equiv 2 k(\bmod \operatorname{gcd}(m, n))$. Consider the following system of simultaneous congruences 


$$
\begin{aligned}
& l \equiv k \quad(\bmod m), \\
& l \equiv-k+s_{1}+s_{2} \quad(\bmod n) .
\end{aligned}
$$

By Theorem 1, there exists a solution $l$. Hence $\left[k, k-s_{1}\right]=\left[l,-l+s_{2}\right]$, which means that $L_{s_{1}} \cap L_{s_{2}}^{-} \neq \varnothing$. It is easy to see that this contradicts the fact that $\left|V_{30}\right|=0$.

(b) For all $k$ we have $s_{1}+s_{2} \equiv 2 k+1(\bmod \operatorname{gcd}(m, n))$. Consider the following system of simultaneous congruences for some fixed $k$

$$
\begin{aligned}
& l \equiv k \quad(\bmod m), \\
& l \equiv-k+s_{1}+s_{2}-1 \quad(\bmod n) .
\end{aligned}
$$

By Theorem 1, there exists a solution $l$. Hence $\left[l,-l+s_{2}-1\right]=\left[k, k-s_{1}\right] \in$ $L_{s_{1}} \subset V_{1}$. Since vertices $\left[l,-l+s_{2}-1\right]$ and $\left[l,-l+s_{2}\right] \in L_{s_{2}}^{-} \subset V_{1}$ are adjacent, it contradicts the fact that $\left|E_{1}\right|=0$.

Thus $f$ is either positive or negative.

Lemma 3 Let $f$ be a 2-rainbow dominating function of $C_{m} \square C_{n}$ such that $w(f)=\frac{m n}{3}$ then

1. $\operatorname{lcm}(m, n) \equiv 0(\bmod 2)$,

2. $\operatorname{gcd}(m, n) \equiv 0(\bmod 3)$.

Proof Let $f$ be a 2-rainbow dominating function of $C_{m} \square C_{n}$ such that $w(f)=\frac{m n}{3}$. By Lemma 2, $f$ is either positive or negative. Assume, without loss of generality, that $f$ is positive. Take any vertex $(i, j) \in C_{m} \square C_{n}$ such that $(i, j) \in V_{1}$. Hence $L_{\llbracket i-j \rrbracket} \subset V_{1}$.

The same argument as in the proof of Lemma 2 shows that

$$
f((i, j))=f([i+2, j+2]) \quad \text { and } \quad f((i, j)) \neq f([i+1, j+1]) .
$$

Hence by induction we have $\left|L_{[[i-j]]}\right| \equiv 0(\bmod 2)$. This together with Theorem 2 proves (1).

Now we will prove (2). If $\operatorname{gcd}(m, n)=1$, then by Corollary 2(3) we have $L_{0}=$ $V\left(C_{m} \square C_{n}\right)$. Since $f$ is positive, we have $L_{0}=V_{1}$. Therefore, $w(f)=m n$. This contradicts our assumption that $w(f)=\frac{m n}{3}$. Consequently $\operatorname{gcd}(m, n)>1$. Suppose that $\operatorname{gcd}(m, n)=2$. Then $(i, j),[i+1, j+1],[i+2, j] \in L_{\llbracket i-j \rrbracket} \subset V_{1}$. This implies that $[i+1, j] \in V_{30}$. However, this contradicts the fact that $\left|V_{30}\right|=0$. Hence $\operatorname{gcd}(m, n) \geq 3$. Assume now that $\operatorname{gcd}(m, n)>3$.

By Corollary 2 and inclusions (1), (2) we get

$$
L_{\llbracket i-j \rrbracket} \subset V_{1}, \quad L_{\llbracket i-j+1 \rrbracket} \subset V_{0}, \quad L_{\llbracket i-j+2 \rrbracket} \subset V_{0} .
$$

In particular $[i+1, j],[i+2, j+1],[i+2, j] \in V_{0}$. To dominate $[i+2, j]$ we must have $[i+3, j] \in V_{1}$, and consequently $L_{\llbracket i-j+3 \rrbracket} \subset V_{1}$. Continuing in this way we get that for any $l \geq 1$ we have

$$
L_{\llbracket i-j+3 l \rrbracket} \subset V_{1}, \quad L_{\llbracket i-j+3 l+1 \rrbracket} \subset V_{0}, \quad L_{\llbracket i-j+3 l+2 \rrbracket} \subset V_{0} .
$$


To prove (2) we must eliminate the following two possibilities.

(a) Let $\operatorname{gcd}(m, n)=3 k+1$ for some $k \geq 1$. Now $L_{\llbracket i-j+3 k \rrbracket} \subset V_{1}$ and $L_{\llbracket i-j+3 k+1 \rrbracket}=$ $L_{\llbracket i-j \rrbracket} \subset V_{1}$. This contradicts (3).

(b) Let $\operatorname{gcd}(m, n)=3 k+2$ for some $k \geq 1$. Now $L_{\llbracket i-j+3 k \rrbracket} \subset V_{1}, L_{\llbracket i-j+3 k+1 \rrbracket} \subset$ $V_{0}$ and $L_{\llbracket i-j+3 k+2 \rrbracket}=L_{\llbracket i-j \rrbracket} \subset V_{1}$. This contradicts (3).

Lemma 4 For $k, l \geq 1$

$$
\gamma_{r 2}\left(C_{6 k} \square C_{3 l}\right)=\gamma_{r 2}\left(C_{3 l} \square C_{6 k}\right)=\frac{6 k \cdot 3 l}{3} .
$$

Proof By Lemma 1, we have $\gamma_{r_{2}}\left(C_{m} \square C_{n}\right) \geq \frac{m n}{3}$. Hence for the proof it suffices to find a $2 \mathrm{RDF}$ of $C_{6 k} \square C_{3 l}$ of weight $\frac{6 k 3 l}{3}$. First we define $f: V\left(C_{6} \square C_{3}\right) \rightarrow \mathscr{P}(\{1,2\})$ as follows

$\begin{array}{cccccc}\varnothing & \varnothing & \{1\} & \varnothing & \varnothing & \{2\} \\ \varnothing & \{2\} & \varnothing & \varnothing & \{1\} & \varnothing \\ \{1\} & \varnothing & \varnothing & \{2\} & \varnothing & \varnothing\end{array}$

It is easy to see that $f$ is a $2 \mathrm{RDF}$ of $C_{6} \square C_{3}$ of weight 6 . The required function on $C_{6 k} \square C_{3 l}$ one can construct using this segment. Finally, the equality $\gamma_{r_{2}}\left(C_{6 k} \square C_{3 l}\right)=$ $\gamma_{r_{2}}\left(C_{3 l} \square C_{6 k}\right)$ follows by the symmetry.

We are ready to prove our main result.

Theorem $3 \gamma_{r 2}\left(C_{m} \square C_{n}\right)=\frac{m n}{3}$ if and only if $m=6 k$ and $n=3 l$ or $m=3 k$ and $n=6 l, k, l \geq 1$.

Proof Let $\gamma_{r 2}\left(C_{m} \square C_{n}\right)=\frac{m n}{3}$ and $f$ be a $2 R D F$ of $C_{m} \square C_{n}$ such that $w(f)=\frac{m n}{3}$. By Lemma 3, we have $\operatorname{gcd}(m, n) \equiv 0(\bmod 3)$ and $\operatorname{lcm}(m, n) \equiv 0(\bmod 2)$. Hence $m \equiv 0(\bmod 3), n \equiv 0(\bmod 3)$ and at least one of $m$ and $n$ is even. This together with Lemma 4 proves our theorem.

The following theorem is the consequence of our considerations.

Theorem 4 Let $m=6 k, n=3 l$. There are $6 \cdot 2^{\frac{\operatorname{gcd}(m, n)}{3}} \gamma_{r 2}$-functions of $C_{m} \square C_{n}$ and $2^{\frac{\operatorname{gcd}(m, n)}{3}} \gamma_{r 2}$-functions up to translations.

Open Access This article is distributed under the terms of the Creative Commons Attribution License which permits any use, distribution, and reproduction in any medium, provided the original author(s) and the source are credited.

\section{References}

Brešar B, Šumenjak TK (2007) Note on the 2-rainbow domination in graphs. Discret Appl Math 155:23942400

Brešar B, Henning MA, Rall DF (2008) Rainbow domination in graphs. Taiwan J Math 12(1):213-225 
Diestel R (1997) Graph theory. Springer-Verlag, New York

Hartnell B, Rall DF (1998) Domination in Cartesian products: Vizing's conjecture. In: Haynes TW, Hedetniemi ST, Slater PJ (eds) Domination in graphs: advanced topics. Marcel Dekker, New York, pp 163-189

Haynes TW, Hedetniemi ST, Slater PJ (1998) Fundamentals of domination in graphs. Marcel Dekker, New York

Stępień Z, Zwierzchowski M (2012) 2-Rainbow domination number of Cartesian products: $C_{n} \square C_{3}$ and $C_{n} \square C_{5}$, J Comb Optim. doi:10.1007/s10878-012-9582-8

Šumenjak TK, Rall DF, Tepeh A (2013) Rainbow domination in the lexicographic product of graphs. Discret Appl Math 161:2133-2141. arXiv:1210.0514v2 [math.CO]

Tong C, Lin X, Yang Y, Luo M (2009) 2-Rainbow domination of generalized Petersen graphs $P(n, 2)$. Discret Appl Math 157:1932-1937

Wu Y, Rad NJ (2013) Bounds on the 2-rainbow domination number of graphs. Graphs Comb 29:1125-1133

Wu Y, Xing H (2010) Note on 2-rainbow domination and roman domination in graphs. Appl Math Lett 23:706-709

Xu G (2009) 2-Rainbow domination in generalized Petersen graphs $P(n, 3),$. Discret Appl Math 157:25702573 